\title{
Current state of stem cell-based therapies: an overview
}

\author{
Riham Mohamed Aly ${ }^{1,2}$ \\ ${ }^{1}$ Department of Basic Dental Science, National Research Centre, Cairo, Egypt; ${ }^{2}$ Stem Cell Laboratory, Center of Excellence for Advanced Sciences, \\ National Research Centre, Cairo, Egypt \\ Correspondence to: Riham Mohamed Aly. Department of Basic Dental Science, Oral \& Dental Research Division, National Research Centre, 33 El \\ Buhouth St., Dokki, Cairo 12622, Egypt. Email: Riham.aly@hotmail.com.
}

\begin{abstract}
Recent research reporting successful translation of stem cell therapies to patients have enriched the hope that such regenerative strategies may one day become a treatment for a wide range of vexing diseases. In fact, the past few years witnessed, a rather exponential advancement in clinical trials revolving around stem cell-based therapies. Some of these trials resulted in remarkable impact on various diseases. In this review, the advances and challenges for the development of stem-cell-based therapies are described, with focus on the use of stem cells in dentistry in addition to the advances reached in regenerative treatment modalities in several diseases. The limitations of these treatments and ongoing challenges in the field are also discussed while shedding light on the ethical and regulatory challenges in translating autologous stem cellbased interventions, into safe and effective therapies.
\end{abstract}

Keywords: Stem cells; therapies; clinical trials; translation

Received: 03 January 2020; Accepted: 30 April 2020; Published: 15 May 2020.

doi: $10.21037 /$ sci-2020-001

View this article at: http://dx.doi.org/10.21037/sci-2020-001

\section{Introduction}

Cell-based therapy as a modality of regenerative medicine is considered one of the most promising disciplines in the fields of modern science \& medicine. Such an advanced technology offers endless possibilities for transformative and potentially curative treatments for some of humanities most life threatening diseases. Regenerative medicine is rapidly becoming the next big thing in health care with the particular aim of repairing and possibly replacing diseased cells, tissues or organs and eventually retrieving normal function. Fortunately, the prospect of regenerative medicine as an alternative to conventional drug-based therapies is becoming a tangible reality by the day owing to the vigorous commitment of the research communities in studying the potential applications across a wide range of diseases like neurodegenerative diseases and diabetes, among many others (1).

Recent research reporting successful translation of stem cell therapies to patients have enriched the hope that such regenerative strategies may one day become a treatment for a wide range of vexing diseases (2). In fact, the past few years witnessed, a rather exponential advancement in clinical trials revolving around stem cell-based therapies. Some of these trials resulted in remarkable impact on various diseases (3). For example, a case of Epidermolysis Bullosa manifested signs of skin recovery after treatment with keratinocyte cultures of epidermal stem cells (4). Also, a major improvement in eyesight of patients suffering from macular degeneration was reported after transplantation of patient-derived induced pluripotent stem cells (iPSCs) that were induced to differentiate into pigment epithelial cells of the retina (5).

However, in spite of the increased amount of publications reporting successful cases of stem cell-based therapies, a major number of clinical trials have not yet acquired full regulatory approvals for validation as stem cell therapies. To date, the most established stem cell treatment is bone marrow transplants to treat blood and immune system disorders $(1,6,7)$.

In this review, the advances and challenges for the development of stem-cell-based therapies are described, with focus on the use of stem cells in dentistry in addition to the advances reached in regenerative treatment modalities 
in several diseases. The limitations of these treatments and ongoing challenges in the field are also discussed while shedding light on the ethical and regulatory challenges in translating autologous stem cell-based interventions, into safe and effective therapies.

\section{Stem cell-based therapies}

Stem cell-based therapies are defined as any treatment for a disease or a medical condition that fundamentally involves the use of any type of viable human stem cells including embryonic stem cells (ESCs), iPSCs and adult stem cells for autologous and allogeneic therapies (8). Stem cells offer the perfect solution when there is a need for tissue and organ transplantation through their ability to differentiate into the specific cell types that are required for repair of diseased tissues.

However, the complexity of stem cell-based therapies often leads researchers to search for stable, safe and easily accessible stem cells source that has the potential to differentiate into several lineages. Thus, it is of utmost importance to carefully select the type of stem cells that is suitable for clinical application $(7,9)$.

\section{Stem cells hierarchy}

There are mainly three types of stem cells. All three of them share the significant property of self-renewal in addition to a unique ability to differentiate. However, it should be noted that stem cells are not homogeneous, but rather exist in a developmental hierarchy (10). The most basic and undeveloped of stem cells are the totipotent stem cells. These cells are capable of developing into a complete embryo while forming the extra-embryonic tissue at the same time. This unique property is brief and starts with the fertilization of the ovum and ends when the embryo reaches the four to eight cells stage. Following that cells undergo subsequent divisions until reaching the blastocyst stage where they lose their totipotency property and assume a pluripotent identity where cells are only capable of differentiating into every embryonic germ layer (ectoderm, mesoderm and endoderm). Cells of this stage are termed "embryonic stem cells" and are obtained by isolation from the inner cell mass of the blastocyst in a process that involves the destruction of the forming embryo. After consecutive divisions, the property of pluripotency is lost and the differentiation capability becomes more lineage restricted where the cells become multipotent meaning that they can only differentiate into limited types of cells related to the tissue of origin. This is the property of "adult stem cells", which helps create a state of homeostasis throughout the lifetime of the organism. Adult stem cells are present in a metabolically quiescent state in almost all specialized tissues of the body, which includes bone marrow and oral and dental tissues among many others (11).

Many authors consider adult stem cells the gold standard in stem cell-based therapies $(12,13)$. Adult stem cells demonstrated signs of clinical success especially in hematopoietic transplants $(14,15)$. In contrast to ESCs, adult stem cells are not subjected to controversial views regarding their origin. The fact that ESCs derivation involves destruction of human embryos renders them unacceptable for a significant proportion of the population for ethical and religious convictions (16-18).

\section{Turning point in stem cell research}

It was in 2006 when Shinya Yamanka achieved a scientific breakthrough in stem cell research by succeeding in generating cells that have the same properties and genetic profile of ESCs. This was achieved via the transient overexpression of a cocktail of four transcription factors; OCT4, SOX2, KLF4 and MYC in, fully differentiated somatic cells, namely fibroblasts $(19,20)$. These cells were called iPSCs and has transformed the field of stem cell research ever since (21). The most important feature of these cells is their ability to differentiate into any of the germ layers just like ESCs precluding the ethical debate surrounding their use. The development of iPSCs technology has created an innovative way to both identify and treat diseases. Since they can be generated from the patient's own cells, iPSCs thus present a promising potential for the production of pluripotent derived patient-matched cells that could be used for autologous transplantation. True these cells symbolize a paradigm shift since they enable researchers to directly observe and treat relevant patient cells; nevertheless, a number of challenges still need to be addressed before iPSCs-derived cells can be applied in cell therapies. Such challenges include; the detection and removal of incompletely differentiated cells, addressing the genomic and epigenetic alterations in the generated cells and overcoming the tumorigenicity of these cells that could arise on transplantation (22). 


\section{Therapeutic translation of stem cell research}

With the rapid increase witnessed in stem cell basic research over the past years, the relatively new research discipline "Translational Research" has evolved significantly building up on the outcomes of basic research in order to develop new therapies. The clinical translation pathway starts after acquiring the suitable regulatory approvals. The importance of translational research lies in it's a role as a filter to ensure that only safe and effective therapies reach the clinic (23). It bridges the gap from bench to bed. Currently, some stem cell-based therapies utilizing adult stem cells are clinically available and mainly include bone marrow transplants of hematopoietic stem cells and skin grafts for severe burns (23). To date, there are more than 3,000 trials involving the use of adult stem cells registered in WHO International Clinical Trials Registry. Additionally, initial trials involving the new and appealing iPSCs based therapies are also registered. In fact, the first clinical attempt employing iPSCs reported successful results in treating macular degeneration (24). Given the relative immaturity in the field of cellular therapy, the outcomes of such trials shall facilitate the understanding of the timeframes needed to achieve successful therapies and help in better understanding of the diseases. However, it is noteworthy that evaluation of stem cell-based therapies is not an easy task since transplantation of cells is ectopic and may result in tumor formation and other complications. This accounts for the variations in the results reported from previous reports. The following section discusses the published data of some of the most important clinical trials involving the use of different types of stem cells both in medicine and in dentistry.

\section{Stem cell-based therapy for neurodegenerative diseases}

The successful generation of neural cells from stem cells in vitro paved the way for the current stem cell-based clinical trials targeting neurodegenerative diseases $(25,26)$. These therapies do not just target detaining the progression of irrecoverable neuro-degenerative diseases like Parkinson's, Alzheimer's, amyotrophic lateral sclerosis (ALS), and multiple sclerosis (MS), but are also focused on completely treating such disorders.

\section{Parkinson's disease (PD)}

PD is characterized by a rapid loss of midbrain dopaminergic neurons. The first attempt for using human ESC cells to treat PD was via the generation of dopaminergic-like neurons, later human iPSCs was proposed as an alternative to overcome ESCs controversies (27). Both cells presented hope for obtaining an endless source of dopaminergic neurons instead of the previously used fetal brain tissues. Subsequently, protocols that mimicked the development of dopaminergic neurons succeeded in generating dopaminergic neurons similar to that of the midbrain which were able to survive, integrate and functionally mature in animal models of PD preclinically (28). Based on the research presented by different groups; the "Parkinson's Global Force" was formed which aimed at guiding researchers to optimize their cell characterization and help promote the clinical progress toward successful therapy. Recently, In August 2018, Shinya Yamanka initiated the first approved clinical trial to treat PD using iPSCs. Seven patients suffering from moderate PD were recruited (29). Donor matched allogeneic cells were used to avoid any genetic influence of the disease. The strategy behind the trial involved the generation of dopaminergic progenitors followed by surgical transplantation into the brains of patients by a special device. In addition, immunosuppressant medications were given to avoid any adverse reaction. Preliminary results so far revealed the safety of the treatment.

\section{MS}

MS is an inflammatory and neurodegenerative autoimmune disease of the central nervous system. Stem cell-based therapies are now exploring the possibility of halting the disease progression and reverse the neural damage. A registered phase 1 clinical trial was conducted by the company Celgene ${ }^{\mathrm{TM}}$ in 2014 using placental-derived mesenchymal stem cells (MSCs) infusion to treat patients suffering from MS (30). This trial was performed at 6 centers in the United States and 2 centers in Canada and included 16 patients. Results demonstrated that cellular infusions were safe with no signs of paradoxical aggravation. However, clinical responses from patients indicated that the cellular treatment did not improve the MS condition (31). For the last decade immunoablative therapy demonstrated accumulative evidence of inducing long-term remission and improvement of disability caused by MS. This approach involves the replacement of the diseased immune system through administration of high-dose immunosuppressive therapy followed by hematopoietic stem cells infusion (32). However, immunoablation strategies demonstrated 
several complications such as infertility and neurological disabilities. A number of randomized controlled trials are planned to address these concerns (32). Currently, new and innovative stem cell-based therapies for MS are only in the initial stages, and are based on different mechanisms exploring the possibility of replacing damaged neuronal tissue with neural cells derived from iPSCs however, the therapeutic potential of iPSCs is still under research (33).

\section{$A L S$}

ALS is a neurodegenerative disease that causes degeneration of the motor neurons which results in disturbance in muscle performance. The first attempt to treat ALS was through the transplantation of MSCs in a mouse model. The outcomes of this experiment were promising and resulted in a decrease of the disease manifestations and thus providing proof of principal (34). Based on these results, several planned/ongoing clinical trials are on the way. These trials mainly assess the safety of the proposed concept and have not proved clinical success to date. Notably, while pre-clinical studies have reported that cells derived from un-diseased individuals are superior to cells from ALS patients; most of the clinical trials attempted have employed autologous transplantation. This information may account for the absence of therapeutic improvement reported (35).

\section{Spinal cord injury}

Other neurologic indications for the use of stem cells are spinal cord injuries. Though the transplantation of different forms of neural stem cells and oligo-dendrocyte progenitors has led to growth in the axons in addition to neural connectivity which presents a possibility for repair (36), proof of recovered function has yet to be established in stringent clinical trials. Nevertheless, Japan has recently given approval to stem-cell treatment for spinal-cord injuries. This approval was based on clinical trials that are yet to be published and involves 13 patients, who are suffering from recent spinal-cord injury. The Japanese team discovered that injection of stem cells isolated from the patients' bone marrow aided in regaining some lost sensation and mobility. This is the first stem cell-based therapy targeting spinal-cord injuries to gain governmental approval to offer to patients (37).

\section{Stem cell-based therapies for ocular diseases}

A huge number of the currently registered clinical trials for stem cell-based therapies target ocular diseases. This is mainly due to the fact that the eye is an immune privileged site. Most of these trials span various countries including Japan, China, Israel, Korea, UK, and USA and implement allogeneic ESC lines $(35,36)$. Notably, the first clinical trial to implement the use autologous iPSCs-derived retinal cells was in Japan which followed the new regulatory laws issued in 2014 by Japan's government to regulate regenerative medicine applications. Two patients were recruited in this trial, the first one received treatment for macular degeneration using iPSCs-generated retinal cell sheet (37). After 1 year of follow-up, there were no signs of serious complications including abnormal proliferation and systemic malignancy. Moreover, there were no signs of rejection of the transplanted retinal epithelial sheet in the second year follow-up. Most importantly, the signs of corrected visual acuity of the treated eye were reported. These results were enough to conclude that iPSCs-based autologous transplantation was safe and feasible (38). It is worthy to mention that the second patient was withdrawn from the study due to detectable genetic variations the patient's iPSCs lines which was not originally present in the patient's original fibroblasts. Such alterations may jeopardize the overall safety of the treatment. The fact that this decision was taken, even though the performed safety assays did not demonstrate tumorgenicity in the iPSCsderived retinal pigment epithelium (RPE) cells, indicates that researchers in the field of iPSCs have full awareness of the importance of safety issues (39).

\section{Stem cell-based therapies for treatment of diabetes}

Pancreatic beta cells are destructed in type 1 diabetes mellitus, because of disorders in the immune system while in type 2 insulin insufficiency is caused by failure of the beta-cell to normally produce insulin. In both cases the affected cell is the beta cell, and since the pancreas does not efficiently regenerate islets from endogenous adult stem cells, other cell sources were tested (38). Pluripotent stem cells (PSCs) are considered the cells of choice for beta cell replacement strategies (39). Currently, there are 
a few industry-sponsored clinical trials that are registered targeting beta cell replacement using ESCs. These trials revolve around the engraftment of insulin-producing beta cells in an encapsulating device subcutaneously to protect the cells from autoimmunity in patients with type 1 diabetes (40). The company ViaCyte ${ }^{\mathrm{TM}}$ in California recently initiated a phase I/II trial (NCT02239354) in 2014 in collaboration with Harvard University. This trial involves 40 patients and employs two subcutaneous capsules of insulin producing beta cells generated from ESCs. The results shall be interesting due to the ease of monitoring and recovery of the transplanted cells. The preclinical studies preceding this trial demonstrated successful glycemic correction and the devices were successfully retrieved after 174 days and contained viable insulin-producing cells (41).

\section{Stem cells in dentistry}

Stem cells have been successfully isolated from human teeth and were studied to test their ability to regenerate dental structures and periodontal tissues. MSCs were reported to be successfully isolated from dental tissues like dental pulp of permanent and deciduous teeth, periodontal ligament, apical papilla and dental follicle (42-44). These cells were described as an excellent cell source owing to their ease of accessibility, their ability to differentiate into osteoblasts and odontoblasts and lack of ethical controversies (45). Moreover, dental stem cells demonstrated superior abilities in immunomodulation properties either through cell to cell interaction or via a paracrine effect (46). Stem cells of non-dental origin were also suggested for dental tissue and bone regeneration. Different approaches were investigated for achieving dental and periodontal regeneration (47); however, assessments of stem cells after transplantation still require extensive studying. Clinical trials have only recently begun and their results are yet to be fully evaluated. However, by carefully applying the knowledge acquired from the extensive basic research in dental and periodontal regeneration, stem cell-based dental and periodontal regeneration may soon be a readily available treatment. To date, there are more than 6,000 clinical trials involving the use of with stem cells, however only a total of 44 registered clinical trials address oral diseases worldwide (48). Stem cell-based clinical trials with reported results targeting the treatment of oral disease are discussed below.

\section{Dental pulp regeneration}

The first human clinical study using autologous dental pulp stem cells (DPSCs) for complete pulp regeneration was reported by Nakashima et al. in 2017 (49). This pilot study was based on extensive preclinical studies conducted by the same group (50). Patients with irreversible pulpitis were recruited and followed up for 6 months following DPSCs' transplantation. Granulocyte colony-stimulating factor was administered to induce stem cell mobilization to enrich the stem cell populations. The research team reported that the use of DPSCs seeded on collagen scaffold in molars and premolars undergoing pulpectomy was safe. No adverse events or toxicity were demonstrated in the clinical and laboratory evaluations. Positive electric pulp testing was obtained after cell transplantation in all patients. Moreover, magnetic resonance imaging of the de-novo tissues formed in the root canal demonstrated similar results to normal pulp, which indicated successful pulp regeneration. A different group conducted a clinical trial that recruited patients diagnosed with necrotic pulp. Autologous stem cells from deciduous teeth were employed to induce pulp regeneration (51). Follow-up of the cases after a year from the intervention reported evidence of pulp regeneration with vascular supply and innervation. In addition, no signs of adverse effects were observed in patients receiving DPSCs transplantation. Both trials are proceeding with the next phases, however the results obtained are promising.

\section{Periodontal tissue regeneration}

Aimetti et al. performed a study which included eleven patients suffering from chronic periodontitis and have one deep intra bony defect in addition to the presence of one vital tooth that needs extraction (52). Pulp tissue was passed through $50-\mu \mathrm{m}$ filters in presence of collagen sponge scaffold and was followed by transplantation in the bony defects caused by periodontal disease. Both clinical and radiographic evaluations confirmed the efficacy of this therapeutic intervention. Periodontal examination, attachment level, and probe depth showed improved results in addition to significant stability of the gingival margin. 
Moreover, radiographic analysis demonstrated bone regeneration.

\section{Regeneration of mandibular bony defects}

The first clinical study using DPSCs for oro-maxillo-facial bone regeneration was conducted in 2009 (53). Patients in this study suffered from extreme bone loss following extraction of third molars. A bio-complex composed of DPSCs cultured on collagen sponge scaffolds was applied to the affected sites. Vertical repair of the damaged area with complete restoration of the periodontal tissue was demonstrated six months after the treatment. Three years later, the same group published a report evaluating the stability and quality of the regenerated bone after DPSCs transplantation (54). Histological and advanced holotomography demonstrated that newly formed bone was uniformly vascularized. However, it was of compact type, rather than a cancellous type which is usually the type of bone in this region.

\section{Stem cells for treatment of Sjögren's syndrome}

Sjögren's syndrome (SS) is a systemic autoimmune disease marked by dry mouth and eyes. A novel therapeutic approach for SS. utilizing the infusion of MSCs in 24 patients was reported by Xu et al. in 2012 (55). The strategy behind this treatment was based on the immunologic regulatory functions of MSCs. Infused MSCs migrated toward the inflammatory sites in a stromal cell-derived factor-1-dependent manner. Results reported from this clinical trial demonstrated suppressed autoimmunity with subsequent restoration of salivary gland secretion in SS patients.

\section{Stem cells and tissue banks}

The ability to bank autologous stem cells at their most potent state for later use is an essential adjuvant to stem cell-based therapies. In order to be considered valid, any novel stem cell-based therapy should be as effective as the routine treatment. Thus, when appraising a type of stem cells for application in cellular therapies, issues like immune rejection must be avoided and at the same time large numbers of stem cells must be readily available before clinical implementation. iPSCs theoretically possess the ability to proliferate unlimitedly which pose them as an attractive source for use in cell-based therapies. Unlike, adult stem cells iPSCs ability to propagate does not decrease with time (22). Recently, California Institute for Regenerative Medicine (CIRM) has inaugurated an iPSCs repository to provide researchers with versatile iPSCs cell lines in order to accelerate stem cell treatments through studying genetic variation and disease modeling. Another important source for stem cells banking is the umbilical cord. Umbilical cord is immediately cryopreserved after birth; which permits stem cells to be successfully stored and ready for use in cell-based therapies for incurable diseases of a given individuals. However, stem cells of human exfoliated deciduous teeth (SHEDs) are more attractive as a source for stem cell banking. These cells have the capacity to differentiate into further cell types than the rest of the adult stem cells (56). Moreover, procedures involving the isolation and cryopreservation of these cells are un-complicated and not aggressive. The most important advantage of banking SHEDs is the insured autologous transplant which avoids the possibility of immune rejection (57). Contrary to cord blood stem cells, SHEDs have the ability to differentiate into connective tissues, neural and dental tissues (58) Finally, the ultimate goal of stem cell banking, is to establish a repository of high-quality stem cell lines derived from many individuals for future use in therapy.

\section{Current regulatory guidelines for stem cell- based therapies}

With the increased number of clinical trials employing stem cells as therapeutic approaches, the need for developing regulatory guidelines and standards to ensure patients safety is becoming more and more essential. However, the fact that stem cell therapy is rather a new domain makes it subject to scientific, ethical and legal controversies that are yet to be regulated. Leading countries in the field have devised guidelines serving that purpose. Recently, the Food and Drug Administration (FDA) has released regulatory guidelines to ensure that these treatments are safe and effective (59). These guidelines state that; treatments involving stem cells that have been minimally manipulated and are intended for homogeneous use do not require premarket approval to come into action and shall only be subjected to regulatory guidelines against disease transmission. In 2014, a radical regulatory reform in Japan occurred with the passing of two new laws that permitted conditional approval of cell-based treatments following early phase clinical trials on the condition that clinical safety data are provided from at least ten patients. 
These laws allow skipping most of the traditional criteria of clinical trials in what was described as "fast track approvals" and treatments were classified according to risk (60). To date, the treatments that acquired conditional approval include those targeting; spinal-cord injury, cardiac disease and limb ischemia (61). Finally, regulatory authorities are now demanding application of standardization and safety regulations protocols for cellular products, which include the use of Xeno-free culture media, recombinant growth factors in addition to "Good Manufacturing Practice" (GMP) culture supplies.

\section{Challenges \& ethical issues facing stem cell- based therapies}

Stem cell-based therapies face many obstacles that need to be urgently addressed. The most persistent concern is the ethical conflict regarding the use of ESCs. As previously mentioned, ESCs are far superior regarding their potency; however, their derivation requires destruction human embryos. True, the discovery of iPSCs overcame this concern; nevertheless, iPSCs themselves currently face another ethical controversy of their own which addresses their unlimited capacity of differentiation with concerns that these cells could one day be applied in human cloning. The use of iPSCs in therapy is still considered a high-risk treatment modality, since transplantation of these cells could induce tumor formation. Such challenge is currently addressed through developing optimized protocols to ensure their safety in addition to developing global clinical-grade iPSCs cell lines before these cells are available for clinical use (61). As for MSCs, these cells have been universally considered safe, however continuous monitoring and prolonged follow-up should be the focus of future research to avoid the possibility of tumor formation after treatments (62). Finally, it could be postulated that one of the most challenging ethical issues faced in the field of stem cell-based therapies at the moment, is the increasing number of clinics offering unproven stem cell-based treatments. Researchers are thus morally obligated to ensure that ethical considerations are not undermined in pursuit of progress in clinical translation.

\section{Conclusions}

Stem cell therapy is becoming a tangible reality by the day, thanks to the mounting research conducted over the past decade. With every research conducted the possibilities of stem cells applications increased in spite of the many challenges faced. Currently, progress in the field of stem cells is very promising with reports of clinical success in treating various diseases like; neurodegenerative diseases and macular degeneration progressing rapidly. iPSCs are conquering the field of stem cells research with endless possibilities of treating diseases using patients own cells. Regeneration of dental and periodontal tissues using MSCs has made its way to the clinic and soon enough will become a valid treatment. Although, challenges might seem daunting, stem cell research is advancing rapidly and cellular therapeutics is soon to be applicable. Fortunately, there are currently tremendous efforts exerted globally towards setting up regulatory guidelines and standards to ensure patients safety. In the near future, stem cell-based therapies shall significantly impact human health.

\section{Acknowledgments}

Funding: None.

\section{Footnote}

Conflicts of Interest: The author has completed the ICMJE uniform disclosure form (available at http://dx. doi. org/10.21037/sci-2020-001). The author has no conflicts of interest to declare.

Ethical Statement: The author is accountable for all aspects of the work in ensuring that questions related to the accuracy or integrity of any part of the work are appropriately investigated and resolved.

Open Access Statement: This is an Open Access article distributed in accordance with the Creative Commons Attribution-NonCommercial-NoDerivs 4.0 International License (CC BY-NC-ND 4.0), which permits the noncommercial replication and distribution of the article with the strict proviso that no changes or edits are made and the original work is properly cited (including links to both the formal publication through the relevant DOI and the license). See: https://creativecommons.org/licenses/by-nc-nd/4.0/.

\section{References}

1. Chari S, Nguyen A, Saxe J. Stem Cells in the Clinic. Cell Stem Cell 2018;22:781-2. 
2. Madl CM, Heilshorn SC, Blau HM. Bioengineering strategies to accelerate stem cell therapeutics. Nature 2018;557:335-42.

3. Pérez López S, Otero Hernández J. Advances in stem cell therapy. Adv Exp Med Biol 2012;741:290-313.

4. Hirsch T, Rothoeft T, Teig N, et al. Regeneration of the entire human epidermis using transgenic stem cells. Nature 2017;551:327-32.

5. Mandai M, Kurimoto Y, Takahashi M. The authors reply. Vol. 377, New England Journal of Medicine. Massachusetts Medical Society, 2017:792-3.

6. Trounson A, McDonald C. Stem Cell Therapies in Clinical Trials: Progress and Challenges. Cell Stem Cell 2015;17:11-22.

7. De Luca M, Aiuti A, Cossu G, et al. Advances in stem cell research and therapeutic development. Nat Cell Biol 2019;21:801-11.

8. Zhang FQ, Jiang JL, Zhang JT, et al. Current status and future prospects of stem cell therapy in Alzheimer's disease. Neural Regen Res 2020;15:242.

9. Bobba S, Di Girolamo N, Munsie M, et al. The current state of stem cell therapy for ocular disease. Exp Eye Res 2018;177:65-75.

10. Gaskell T, Englund MCO, Hyllner J. Human embryonic stem cells. In: Steinhoff G. editor. Regenerative Medicine - from Protocol to Patient: 2. Stem Cell Science and Technology. 3rd edition. Springer, 2016.

11. Clevers H, Watt FM. Defining Adult Stem Cells by Function, not by Phenotype. Annu Rev Biochem 2018;87:1015-27.

12. Prentice DA. Adult Stem Cells. Circ Res 2019;124:837-9.

13. Lukomska B, Stanaszek L, Zuba-Surma E, et al. Challenges and Controversies in Human Mesenchymal Stem Cell Therapy. Stem Cells Int 2019;2019:9628536.

14. Morrison SJ, Scadden DT. The bone marrow niche for haematopoietic stem cells. Nature 2014;505:327-34.

15. Sharpe M, Leoni G, Hyllner J. Stem Cells. In: McQueen CA. Comprehensive Toxicology. 3rd edition. Elsevier, 2017:23-59.

16. Green RM. Ethical Considerations. In: Lanza R, Atala A. editors. Essentials of Stem Cell Biology. 3rd edition. Elsevier, 2013:595-604.

17. Brignier AC, Gewirtz AM. Embryonic and adult stem cell therapy. J Allergy Clin Immunol 2010;125:S336-44.

18. Lo B, Parham L. Ethical issues in stem cell research. Endocr Rev 2009;30:204-13.
19. Takahashi K, Yamanaka S. Induction of Pluripotent Stem Cells from Mouse Embryonic and Adult Fibroblast Cultures by Defined Factors. Cell 2006;126:663-76.

20. Takahashi K, Tanabe K, Ohnuki M, et al. Induction of Pluripotent Stem Cells from Adult Human Fibroblasts by Defined Factors. Cell 2007;131:861-72.

21. Shi Y, Inoue H, Wu JC, et al. Induced pluripotent stem cell technology: a decade of progress. Nat Rev Drug Discov 2017;16:115-30.

22. Attwood SW, Edel MJ. iPS-Cell Technology and the Problem of Genetic Instability-Can It Ever Be Safe for Clinical Use? J Clin Med 2019;8:288.

23. Poulos J. The limited application of stem cells in medicine: a review. Stem Cell Res Ther 2018;9:1.

24. Mandai M, Watanabe A, Kurimoto Y, et al. Autologous induced stem-cell-derived retinal cells for macular degeneration. N Engl J Med 2017;376:1038-46.

25. McGinley LM, Kashlan ON, Bruno ES, et al. Human neural stem cell transplantation improves cognition in a murine model of Alzheimer's disease. Sci Rep 2018;8:14776.

26. Fang Y, Gao T, Zhang B, et al. Recent Advances: Decoding Alzheimer's Disease With Stem Cells. Front Aging Neurosci 2018;10:77.

27. Kikuchi T, Morizane A, Doi D, et al. Human iPS cellderived dopaminergic neurons function in a primate Parkinson's disease model. Nature 2017;548:592-6.

28. Swistowski A, Peng J, Liu Q, et al. Efficient generation of functional dopaminergic neurons from human induced pluripotent stem cells under defined conditions. Stem Cells 2010;28:1893-904.

29. Takahashi J. Stem cell therapy for Parkinson's disease. Expert Rev Neurother 2007;7:667-75.

30. Lublin FD, Bowen JD, Huddlestone J, et al. Human placenta-derived cells(pda-001) for the treatment of adults with multiple sclerosis: Arandomized,placebocontrolled, multiple-dose study. Mult Scler Relat Disord 2014;3:696-704.

31. Cuascut FX, Hutton GJ. Stem Cell-Based Therapies for Multiple Sclerosis: Current Perspectives. Biomedicines 2019;7:26.

32. Delemarre EM, Van Den Broek T, Mijnheer G, et al. Autologous stem cell transplantation AIDS autoimmune patients by functional renewal and TCR diversification of regulatory T cells. Blood 2016;127:91-101.

33. Thiruvalluvan A, Czepiel M, Kap YA, et al. Survival and 
Functionality of Human Induced Pluripotent Stem CellDerived Oligodendrocytes in a Nonhuman Primate Model for Multiple Sclerosis. Stem Cells Transl Med 2016;5:1550-61.

34. Meamar R, Nasr-Esfahani MH, Mousavi SA, et al. Stem cell therapy in amyotrophic lateral sclerosis. J Clin Neurosci 2013;20:1659-63.

35. Mazzini L, Ferrari D, Andjus PR, et al. Advances in stem cell therapy for amyotrophic lateral sclerosis. Expert Opin Biol Ther 2018;18:865-81.

36. Assinck P, Duncan GJ, Hilton BJ, et al. Cell transplantation therapy for spinal cord injury. Nat Neurosci 2017;20:637-47.

37. Cyranoski D. Japan's approval of stem-cell treatment for spinal-cord injury concerns scientists. Nature 2019;565:544-5.

38. Dor Y, Brown J, Martinez OI, et al. Adult pancreatic $\beta$-cells are formed by self-duplication rather than stem-cell differentiation. Nature 2004;429:41-6.

39. Pagliuca FW, Millman JR, Gürtler M, et al. Generation of functional human pancreatic $\beta$ cells in vitro. Cell 2014;159:428-39.

40. Schulz TC, Young HY, Agulnick AD, et al. A scalable system for production of functional pancreatic progenitors from human embryonic stem cells. PLoS One 2012;7:e37004.

41. Vegas AJ, Veiseh O, Gürtler M, et al. Long-term glycemic control using polymer-encapsulated human stem cellderived beta cells in immune-competent mice. Nat Med 2016;22:306-11.

42. Raspini G, Wolff J, Helminen M, et al. Dental Stem Cells Harvested from Third Molars Combined with Bioactive Glass Can Induce Signs of Bone Formation In Vitro. J Oral Maxillofac Res 2018;9:e2.

43. Yasui T, Mabuchi Y, Morikawa S, et al. Isolation of dental pulp stem cells with high osteogenic potential. Inflamm Regen 2017;37:8.

44. Suchánek J, Visek B, Soukup T, et al. Stem cells from human exfoliated deciduous teeth--isolation, long term cultivation and phenotypical analysis. Acta Medica (Hradec Kralove) 2010;53:93-9.

45. Hu L, Liu Y, Wang S. Stem cell-based tooth and periodontal regeneration. Oral Dis 2018;24:696-705.

46. Ramenzoni LL, Russo G, Moccia MD, et al. Periodontal bacterial supernatants modify differentiation, migration and inflammatory cytokine expression in human periodontal ligament stem cells. PLoS One 2019;14:e0219181.

47. Bartold PM, Gronthos S, Ivanovski S, et al. Tissue engineered periodontal products. J Periodontal Res 2016;51:1-15.

48. Yamada Y, Nakamura-Yamada S, Kusano K, et al. Clinical potential and current progress of dental pulp stem cells for various systemic diseases in regenerative medicine: A concise review. Int J Mol Sci 2019;20:1132.

49. Nakashima M, Iohara K, Murakami M, et al. Pulp regeneration by transplantation of dental pulp stem cells in pulpitis: a pilot clinical study. Stem Cell Res Ther 2017;8:61.

50. Nakashima M, Iohara K. Mobilized dental pulp stem cells for pulp regeneration: initiation of clinical trial. J Endod 2014;40:S26-32.

51. Xuan K, Li B, Guo H, et al. Deciduous autologous tooth stem cells regenerate dental pulp after implantation into injured teeth. Sci Transl Med 2018;10:eaaf3227.

52. Aimetti M, Ferrarotti F, Gamba M, et al. Regenerative Treatment of Periodontal Intrabony Defects Using Autologous Dental Pulp Stem Cells: A 1-Year FollowUp Case Series. Int J Periodontics Restorative Dent 2018;38:51-8.

53. d'Aquino R, De Rosa A, Lanza V, et al. Human mandible bone defect repair by the grafting of dental pulp stem/ progenitor cells and collagen sponge biocomplexes. Eur Cell Mater 2009;18:75-83.

54. Giuliani A, Manescu A, Langer M, et al. Three Years After Transplants in Human Mandibles, Histological and In-Line Holotomography Revealed That Stem Cells Regenerated a Compact Rather Than a Spongy Bone: Biological and Clinical Implications. Stem Cells Transl Med 2013;2:316-24.

55. Xu J, Wang D, Liu D, et al. Allogeneic mesenchymal stem cell treatment alleviates experimental and clinical Sjögren syndrome. Blood 2012;120:3142-51.

56. Ma L, Makino Y, Yamaza H, et al. Cryopreserved Dental Pulp Tissues of Exfoliated Deciduous Teeth Is a Feasible Stem Cell Resource for Regenerative Medicine. PLoS One 2012;7:e51777.

57. Arora V, Arora P, Munshi AK. Banking stem cells from human exfoliated deciduous teeth (SHED): saving for the future. J Clin Pediatr Dent 2009;33:289-94.

58. Cordeiro MM, Dong Z, Kaneko T, et al. Dental Pulp Tissue Engineering with Stem Cells from Exfoliated 
Deciduous Teeth. J Endod 2008;34:962-9.

59. Marks PW, Witten CM, Califf RM. Clarifying StemCell Therapy's Benefits and Risks. N Engl J Med 2017;376:1007-9.

60. Konomi K, Tobita M, Kimura K, et al. New Japanese initiatives on stem cell therapies. Cell Stem Cell

doi: 10.21037/sci-2020-001

Cite this article as: Aly RM. Current state of stem cell-based therapies: an overview. Stem Cell Investig 2020;7:8.
2015;16:350-2.

61. Cyranoski D. The potent effects of Japan's stem-cell policies. Nature 2019;573:482-5.

62. Volarevic V, Markovic BS, Gazdic M, et al. Ethical and safety issues of stem cell-based therapy. Int J Med Sci 2018;15:36-45. 